Conclusion This study is the first in the field showing no effect of placebo treatment using sham therapy on newborns opening discussions about the age when placebo effect starts.

\section{AIRWAY SKILLS IN PAEDIATRIC CRITICAL CARE UNITS; A NATIONAL SURVEY OF NON-ANAESTHETIC TRAINEES}

doi:10.1136/archdischild-2012-302724.0965

'F Murray, 'M Vaidya, ${ }^{2 B}$ Clifton. ${ }^{1}$ Paediatric Critical Care; ${ }^{2}$ Paediatric Anaesthesia, Barts Health NHS Trust, London, UK

Background Airway interventions in critically ill patients are associated with a higher incidence of complications; inadequate skill can have serious implications. We conducted a survey to investigate airway skills training of non-anaesthetic trainees working in PICU in the UK and Ireland and to assess the need for an airway skills course specifically for these trainees.

Methods A survey was emailed to PICUs listed on the Paediatric Intensive Care Audit Network requesting completion by nonanaesthetic trainees.

Results Of 25 units eligible, 14 confirmed participation (53\%) Sixty-five trainees responded. Most were of a paediatric background and of ST5 level and above. Only 48\% received airway training on induction to PICU. This comprised of bag-mask ventilation (100\%), airway adjuncts (71\%), laryngeal masks (48\%), intubation (74\%), and emergency cricothyroidotomy (19\%). Previous training came from advanced paediatric life support (APLS), neonatal intensive care, in-house resuscitation training or anaesthetic modules. Emergency airway scenarios were encountered by $90 \%$ and included accidental extubations, blocked or dislodged endotracheal and tracheostomy tubes, respiratory arrests and upper airway obstruction. Of these only $64 \%$ felt they had sufficient training to manage the situation as the first attending doctor. Availability of an airway skills course was supported by $83 \%$. Comments highlighted the importance placed on dedicated theatre time.

Conclusions The percentage of trainees who encountered emergency airway scenarios illustrates the importance of basic airway skills. However only $48 \%$ of trainees received airway training on induction to PICU. The numbers in favour of an airway course could indicate a current gap in training.

\section{THE NONTHYROIDAL ILLNESS SYNDROME IN SEPTIC CHILDREN}

doi:10.1136/archdischild-2012-302724.0966

ML Neamtu, L Dobrota, MB Neamtu, CS Berghea Neamtu, FS Diac. Pediatric Clinic Hospital, Lucian Blaga University of Sibiu, Sibiu, Romania

Backgroud The interpretation of thyroid function tests in the PICU patients can be difficult because thyroid hormone concentrations are affected by nonthyroidial stresses. Most patients fall into sick euthyroid syndrome or nonthyroidal illness syndrome (NTIS). The TH therapy during critical illness remains controversial.

Aim To evaluate the NTIS in septic children.

Method The prospective study conducted on 65 patients admitted in PICU during 12 months period. Inclusion criteria: age $>1$ month, admission for critical illness, treatment with corticosteroids, no (documented) history of thyroid pathology. The patients were divided into 2 groups: sepsis and non-sepsis group (dehydration, poisoning, cardiac failure). All patients received corticosteroids. Some of them received in addition dopamine or presented malnutrition. The thyroid hormone assessment was made after the critical diagnosis was established.

Results Sepsis group: 30 patients. Non-sepsis group: 35 patients. NTIS was strongly correlated with sepsis $(p<0,008)$. Not strongly variation with the addition of dopamine, either for sepsis group $(p<0.07)$ and non-sepsis group $(p<0.03)$. Few patients with malnutrition for both groups, without statistical interpretation. Most frequent variation was observed as "low T3-T4 syndrome" $(63 \%)$. One single patient (6 months old) was diagnosed as critical hypothyroidism (Sandifer syndrome history), based on clinical complains (severe hypothermia - 32.8 Celsius degrees, bradycardia, altered mental status), less conclusive thyroid hormone tests (high $\mathrm{TSH})$, the only one who received $\mathrm{TH}$ therapy.

Conclusion The study confirmed significant correlation between NTIS and sepsis in children (40\%). Reassessment of thyroid function should be done away from critical episode.

\section{DIRECT AND INDIRECT FINANCIAL BURDEN ON THE FAMILIES OF CHILDREN RECEIVING CRITICAL CARE AT TERTIARY LEVEL PICU IN INDIA}

doi:10.1136/archdischild-2012-302724.0967

'V Shukla, 1,2SM Nimbalkar. 'Department of Pediatrics, Pramukhswami Medical College; ${ }^{2}$ Central Research Services, Charutar Arogya Mandal, Anand, India

Background and Aims PICU admissions result in high expenditure and impacts financial dynamics of families. We analyzed direct and indirect costs of Pediatric critical care, differences between patient groups, actual out of pocket expenditure, interventions provided and final outcomes.

Methods 784 children were admitted over 27 months. 518 received discharge, 57 died, 207 discharged against medical advice \& 2 absconded. 116 patients were excluded due to missing details. 668 were analyzed for Average length of stay(ALOS), average hospital expenses(AHE), average hospital expenses/day(AHED), average pharmacy expenses(APE), average pharmacy expenses/day(APED).

Results ALOS in PICU was 6.125 days, AHE was $\$ 231 /$ patient and AHED of $\$ 40 /$ patient/day, APE was $\$ 102.3 /$ patient \& the APED was $\$ 16.7$. Overall expenses were higher in younger patients especially infants. Males had more expense and length of stay. Patients who died had 4.23days(ALOS), \$315(AHE), \$74.5/day(AHED), \$134.8(APE)\& \$32/day(APED); as opposed to those who were discharged 7.21days(ALOS), \$224(AHE), \$31.1/day(AHED), \$97.9(APE)\& \$13.6/day(APED). Uninsured patients had 3.5 times higher expenses than insured (Analysis of expenses uncovered in insurance cover). Ventilated patients had 9.788days(ALOS), \$395.2(AHE), \$40.4/day(AHED), \$217.5(APE) \& \$22.22/day(APED); compared to unventilated ones who had 4.436days(ALOS), \$117.2(AHE), \$26.4/day(AHED), \$48.7(APE)\& \$11/day(APED). Patients with meningoencephalitis, multiple organ dysfunction syndrome \& septicemic shock had 4, 5, 2.5 times higher expenses than average.

Conclusions Younger patients and those with critical illness had higher expenditure. Intensive care life support interventions like ventilation increase the expenses to over four times average. Direct and indirect expenses incurred from critical care lead to further impoverishment of already poor classes.

\section{ROLE OF A REGIONAL RETRIEVAL SERVICE IN PROVIDING SAFE AND SUSTAINABLE CARDIAC SERVICES FOR CHILDREN}

doi:10.1136/archdischild-2012-302724.0968

${ }^{1,2} \mathrm{NK}$ Puppala, ${ }^{1} \mathrm{~K}$ Atanasov, ' $\mathrm{S}$ Ali, ${ }^{1,3 \mathrm{R}}$ Phatak, 'K Parkins. 'Paediatric Intensive Care, North West and North UK Paediatric Transport Service, Warrington; ${ }^{2}$ Paediatric Intensive Care, Central Manchester University Hospitals NHS Foundation Trust, Manchester, ${ }^{3}$ Paediatric Intensive Care, AlderHey Children's NHS Foundation Trust, Liverpool, UK

Background and Aim Safe and sustainable review of cardiac services for children in England has proposed options for reconfiguration of services. We reviewed the current management of cardiac emergencies at referring hospitals and the role of regional transport team. North West and North Wales Paediatric Transport service 
(NWTS) provides specialist retrieval service across the region. Patients are transferred to Alder Hey Children's hospital (AHCH), a cardiac surgical centre and Royal Manchester Children's hospital (RMCH) a cardiology centre. This review was undertaken to quantify current referral patterns and bed management.

Methods Retrospective review of NWTS retrieval of cardiac patients over the last 18 months.

Results

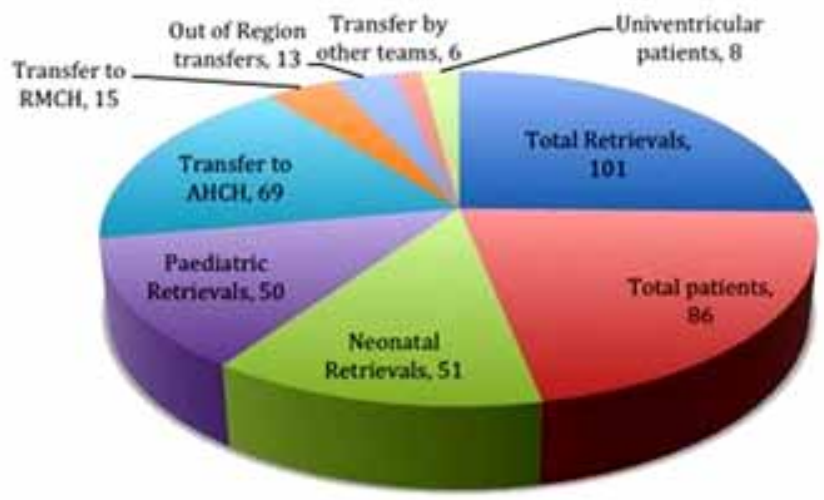

Abstract 968 Figure 1 Results

Abstract 968 Table 1 INTERVENTIONS

\begin{tabular}{lcc}
\hline INTERVENTIONS & REFERRING HOSPITAL & NWTS \\
\hline INTUBATION & 57 & 9 \\
DINOPROSTONE & 29 & 2 \\
INOTROPES & 23 & 10 \\
CENTRAL VENOUS ACCESS & 26 & 16 \\
INHALED NITRIC OXIDE & 0 & 4 \\
\hline
\end{tabular}

Median NWTS retrieval and stabilisation times were 238 and 118 minutes respectively. Only 4 neonates required an additional journey to a regional surgical centre. Total mortality (to discharge home) was $8 / 86 \sim 9.3 \%, 3$ post-operative deaths and 5 offered palliative care (inoperable lesion/other anomalies).

Conclusion For children with congenital heart disease, a regional specialist paediatric retrieval team can optimise bed management, minimise out of region transfers and ensure that time-critical care is delivered locally, safely and effectively.

\section{UNDER-REPORTED? A REVIEW OF UTILISATION OF THE PAEDIATRIC HIGH DEPENDENCY UNIT AT A DISTRICT GENERAL HOSPITAL}

doi:10.1136/archdischild-2012-302724.0969

'A Sylvan, ${ }^{2} \mathrm{M}$ Mukherjee, ${ }^{1} \mathrm{H}$ Mantle. 'Royal Albert Edward Infirmary; ${ }^{2}$ Paediatrics, Royal Albert Edward Infirmary, Wigan, UK

Background and Aims The 'High Dependency Care for Children' (Department of Health, 2001) report stipulates HDU level of care for all in-patient paediatric units. While the importance of regular audit is undisputed, concerns have been raised regarding the accuracy of data available to the Greater Manchester Paediatric \& Child Health Network (GMP\&CHN) and the consequences for optimal service provision.

This study aims to review and compare the utilisation of the Paediatric HDU at our District General Hospital with data available to the regional network (GMP\&CHN).

Methods Retrospective study of Paediatric HDU admissions between 02/09/2009 and 20/11/2011. Data was separated into 12 diagnostic categories and analysed for admission/readmission frequency and length of stay (defined as presence in HDU per calendar day) and compared with the respective figures of the GMP\&CHN 2011 regional audit of Paediatric HDU.

Results 208 patients were admitted 245 times, of which $39(18.75 \%)$ were readmissions. Mean length of stay per admission was 2.28 days (median: 2 days, range: 1-16 days). HDU occupancy rate over the study period (1,014 days) was $55.13 \%$ (559 days). For the period January-August 2011, GMP\&CHN figures show 23 admissions, a $40.35 \%$ underestimation of the actual figure ( 57 admissions).

Conclusions Paediatric HDU activity at our DGH has been underreported. This may not be an uncommon phenomenon nationwide.

We call for further review of HDU activity at units both regionally and nationally with a move towards agreed standards for data dissemination and reporting as per the Paediatric Critical Care Minimum Data Set.

\section{IMPROVING SPECIFICITY OF CLINICAL EVENT DETECTION IN THE NEONATAL INTENSIVE CARE UNIT (NICU)}

doi:10.1136/archdischild-2012-302724.0970

'V Gangadharan, ${ }^{2} \mathrm{~S}$ Mitra, ${ }^{2} \mathrm{H}$ O'Reilly, ${ }^{2} \mathrm{~T}$ Austin, ${ }^{1} \mathrm{AP}$ Gibson. ${ }^{1}$ Medical Physics and Bioengineering, University College London, London; ${ }^{2}$ Cambridge University Hospitals NHS Foundation Trust, Cambridge, UK

Background and Aims Continuous physiological parameter monitoring is routine in NICU. However, high false alarm rates exist that can lead to inappropriate responses from clinical staff.

At present, parameters are assessed independently to generate alarms. The positive predictive value can be increased in adult patients by combining the physiological parameters using statistical models ${ }^{1}$.

We have developed a multi-parameter model, designed for sick newborn infants, which produces alarms based on an integrated assessment of patient physiology. We hypothesized that this model would have greater specificity than conventional single channel alerts.

Methods Continuous physiological data (heart rate, respiration rate, oxygen saturation, blood pressure and temperature) were collected from 6 preterm infants, median gestation 26.1 weeks (range 24.3-28.9). The median period of data collection was 16.5 days (range 10.9-23.2). A mathematical model was developed using Matlab, which 'learnt' 1 hour of normal data in order to subsequently identify abnormal events in the remaining dataset. Adverse clinical events were identified retrospectively from patient notes. Results

Abstract 970 Table 1 Table comparing detection performance

\begin{tabular}{lccc}
\hline & Sensitivity & Specificity & $\begin{array}{c}\text { False positives } \\
\text { per hour }\end{array}$ \\
\hline Existing single-channel alert method & $100 \%$ & $0 \%$ & 1.8 \\
Proposed multi-parameter model & $99 \%$ & $17 \%$ & 0.1 \\
\hline
\end{tabular}

157 clinical events were obtained for analysis. The proposed model increases specificity of event detection, reduces false positives by a factor of 18 and maintains high sensitivity.

Conclusions This pilot study demonstrates that combining existing physiological data using a multi-parameter model improves the specificity of adverse event detection in the NICU.

${ }^{1}$ Tarassenko L et al. Br J Anaesth. 2006; 97:64-8.

\begin{tabular}{l}
\hline 971 NEONATOLOGY-OSTEOPATHY (NE-O) STUDY: RCT ON \\
THE EFFECT OF OSTEOPATHIC MANIPULATIVE
\end{tabular} TREATMENT ON LOS

doi:10.1136/archdischild-2012-302724.0971 